\title{
Evaluación funcional de la vía aérea
}

\author{
Dr. José San Martín', Dra. Solange Caussade 2 \\ 1 Instructor Adjunto. Departamento Otorrinolaringología, Escuela de Medicina. Pontificia Universidad Católica de Chile. \\ 2 Profesor Asistente Adjunto. División Pediatría, Escuela de Medicina. Pontificia Universidad Católica de Chile.
}

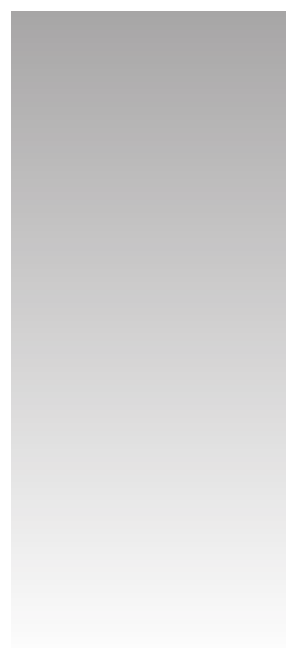

\section{FUNCTIONAL ASESSMENT OF AIRWAY}

Airway function study includes a variety of tests involving various sub-specialties of medicine and related professions. We describe the methods that assess: I) upper airway (nose to larynx): rhinomanometry, acoustic rhinometry and nasopharingolaryngoscopy, 2) central airways (trachea and main bronchi): bronchoscopy, imaging study and Flow / Volume curve, 3) distal or peripheral airway: spirometry, bronchodilator response assessment, airway hyperresponsiveness and measurement of airway resistance (plethysmography, interrupted resistence and impulse oscillometry).

Key words: Airway, functional evaluation.

\section{RESUMEN}

El estudio de la función de la vía aérea abarca una gran variedad de exámenes que implican a diversas subespecialidades de la medicina y profesiones relacionadas. Se describen los métodos que evalúan: I) vía aérea superior (nariz hasta laringe): rinomanometría, rinometría acústica y nasofarigolaringoscopía, 2) vía aérea central (tráquea y bronquios mayores): bronconcoscopia, estudio de imágenes y curva flujo/volumen, 3) vía aérea distal o periférica: espirometría, evaluación de respuesta a broncodilatador y de hiperreactividad bronquial y medición de la resistencia de la vía aérea (pletismografía, resistencia interrumpida y oscilometría de impulso).

Palabras clave: Vía aérea, evaluación funcional.

\section{INTRODUCCIÓN}

La función primordial de la vía aérea es dirigir y acondicionar el aire inspirado. Otras funciones también importantes son la fonación, deglución y protección de los pulmones. La vía aérea se inicia con la nariz, siguen faringe y laringe, luego el árbol traqueo bronquial, con su zona de conducción (tráquea, bronquios mayores, subdivisiones bronquiales hasta bronquiolo terminal), y zona de transición y respiratoria (bronquiolos respiratorios, ductos y sacos alveolares). Su anatomía, histología, su ubicación con respecto a la cavidad torácica determinan las diversas condiciones fisiológicas y patológicas de cada uno de sus segmentos ${ }^{(1,2)}$. Según su ubicación anatómica, en condiciones normales su funcionalidad depende del área de superficie que posee, de la presión atmosférica, presión intrapleural, volumen pulmonar, retracción elástica pulmonar, y en condiciones patológicas influirán la presencia de obstrucción mecánica (pólipos nasales, hipertrofia adenoidea, inflamación nasal, compresiones extrínsecas, o lesiones intrínsecas, etc), inflamación bronquial (asma, fibrosis quística, etc), o de obstrucción dinámica como ocurre en la traqueo y/o broncomalacia. La sintomatología producida por las enfermedades que afectan la vía aérea será clave para sospechar la presencia de una alteración en su función; los síntomas referidos pueden ser estridor, roncopatía, respira-

Correspondencia: Dra Solange Caussade

e-mail: mcaussa@med.puc.cl

ISSN 07|8-332I Derechos reservados. ción bucal, alteraciones de la voz, disfagia,tos persistente con o sin factores gatillantes, sibilancias, hipersecreción bronquial persistente, etc.

En este capítulo se describirán las características fisiológicas y los métodos actuales para evaluar la función de la vía aérea, según su localización: vía aérea superior (nariz hasta laringe), vía aérea central (tráquea y bronquios mayores) y vía aérea pequeña.

\section{ANATOMÍA FUNCIONAL}

\section{Nariz}

Aunque es posible respirar a través de la boca, es la nariz la que anatómica y fisiológicamente está diseñada para hacerlo. Todas las paredes nasales son óseas y están cubiertas por mucosa de tipo respiratorio. La nariz es el segmento de la vía aérea encargado de acondicionar el aire antes de su llegada a los pulmones, aportando al aire inspirado una temperatura y humedad óptimas para que el intercambio gaseoso a nivel pulmonar sea lo más eficiente posible. Para esto son fundamentales los cornetes nasales, estructuras altamente vascularizadas. La mucosa nasal produce mucus en forma constante, logrando un ambiente húmedo que optimiza el aire inspirado $y$, por otro lado, actúa como filtro atrapando algunas partículas en suspensión ${ }^{(1)}$.

\section{Faringe}

La faringe es un segmento compartido por la vía aérea y por el tubo digestivo. Sus paredes son blandas, conformadas fundamentalmente por los músculos constrictores de la 
faringe y la base de la lengua. Esta característica hace que la faringe, a diferencia del resto de la vía aérea, sea colapsable. Para evitar el colapso dinámico durante la inspiración, el tono muscular resulta fundamental, al igual que la acción de algunos músculos que favorecen su dilatación, como el geniogloso, que lleva la base de la lengua hacia delante aumentando el diámetro anteroposterior a nivel orofaríngeo. Durante el sueño, el tono muscular y la acción de los músculos dilatadores disminuyen considerablemente, favoreciendo la disminución del diámetro, lo que a veces llega al colapso de la misma, produciendo ronquidos e incluso apneas obstructivas ${ }^{(1)}$.

Tanto a nivel de la rinofaringe como de la orofaringe existe tejido linfático formando el anillo de Waldeyer. Parte de este anillo corresponde a los adenoides en la rinofaringe y las amígdalas palatinas en la orofaringe. Estas estructuras están presentes en condiciones normales en todos los seres humanos.

\section{Laringe}

Este segmento de la vía aérea es quizás el de funcionamiento más complejo y en él participan gran cantidad de circuitos neuronales sensitivos y motores que deben ser capaces de coordinar una adecuada respiración con una deglución segura y efectiva, además de ser capaces de lograr una buena fonación y proteger la vía aérea. La motilidad cordal (grado de apertura o cierre, tensión) es determinante para la mantención de estos mecanismos.

Al momento de respirar, las cuerdas vocales se abren permitiendo el libre paso del aire a través de la glotis. Al deglutir, la laringe completa asciende, la epiglotis se dobla hacia posterior y las cuerdas vocales se cierran, para evitar la penetración del alimento a la laringe y su aspiración a la tráquea. Al fonar, las cuerdas vocales se cierran y vibran con el paso de aire forzado a través de la glotis; la intensidad y el tono del sonido emitido dependerán de la fuerza con la cual pasa el aire a través de la glotis y de la tensión y grosor de las cuerdas vocales. Durante la primera fase del mecanismo de la tos, las cuerdas vocales se cierran mientras aumenta la presión intratorácica y luego se abren abruptamente para permitir la espiración a alto flujo(l).

\section{Tráquea y bronquios}

La tráquea es un tubo fibromuscular cuyo soporte está constituido por cartílago en forma de C, en la zona ventrolateral, y dorsalmente se completa con músculo liso. Esta conformación se mantiene en los bronquios mayores, y en la medida que los bronquios se van subdividiendo los anillos van siendo reemplazados por placas cartilaginosas de formas irregulares.

Durante el ciclo respiratorio el diámetro del lumen traqueal depende de su ubicación en relación al tórax; en el segmento cervical de la tráquea, extratorácico, este disminuye durante la inspiración ya que la presión atmosférica supera la presión dentro de la vía aérea. El segmento intratorácico disminuye su calibre durante la espiración, debido a que la presión intrapleural es mayor que la existente dentro de la vía aérea. Esto mismo ocurre a nivel bronquial.

A partir de la $12^{\mathrm{a}}$ generación bronquial ya no hay cartílago que sostenga la vía aérea, lo que determina mayor susceptibilidad al colapso. El grado de este último va a depender principalmente de la presión transmural. Las fibras de músculo liso están bajo el control de fibras eferentes del sistema nervioso autónomo, su estimulación colinérgica parasimpática causa contracción, y la estimulación adrenérgica simpática su dilatación, mediada por receptores $\beta 2$, regulándose así el tono y calibre de la luz bronquia| $\left.\right|^{(1,2)}$.

\section{MÉTODOS DE EVALUACIÓN}

\section{Vía áerea superior}

\section{Rinomanometría}

Este examen permite objetivar la obstrucción nasal. Existen distintos tipos de rinomanometría (RMM), siendo la RMM anterior activa (RMMAA) la más frecuentemente utilizada. Esta evalúa el flujo nasal durante la inspiración y la espiración detectando posibles obstrucciones y/o resistencias. Se puede realizar usando una mascarilla facial o bien colocando una oliva en cada narina; el primer dispositivo tiene la ventaja de no deformar las narinas, reduciendo la posibilidad de fuga aérea. Sin embargo, tiene el inconveniente de requerir una importante colaboración del paciente y de no poder realizarse si existe una oclusión total de una fosa nasal o una perforación septal. Luego de colocada la mascarilla, los flujos de aire se miden con el rinomanómetro y los datos se analizan computacionalmente para posteriormente graficarlos en curvas de presión/ volumen. Después de una primera medición en estado basal, el registro se repite bajo el efecto de un vasoconstrictor tópico, lo que permitirá diferenciar entre obstrucciones mecánicas (las que no varían con el vasoconstrictor), vasomotoras (las que mejoran por completo con el vasoconstrictor) y mixtas (la que mejoran parcialmente con el vasoconstrictor). En general, cualquier causa de obstrucción con componentes óseos, cartilaginosos o de tejidos con poco edema o no "vasocontraíbles" mostrará curvas mecánicas, así como etiologías inflamatorias, con edema y tejido susceptible de vasoconstricción nos darán curvas vasomotoras. La patología más representativa de la obstrucción mecánica es la desviación del tabique nasal, así como la más representativa de la obstrucción vasomotora es la hipertrofia de los cornetes inferiores ${ }^{(3)}$.

\section{Rinometría acústica}

Consiste en el estudio de la geometría de la cavidad nasal. Se basa en el análisis de la reflexión del sonido y proporciona un cálculo de áreas de sección transversal de la fosa nasal y de determinados volúmenes nasales. Consiste en aplicar un sonido audible en la ventana nasal mediante un adaptador, con el cuidado de no deformar el vestíbulo nasal. La onda sonora penetra en las fosas y se refleja en las distintas estructuras nasales o en las irregularidades presentes. Se miden las señales de las ondas incidentes y las reflejadas en función del tiempo, lo que permite determinar la distancia respecto de la ventana nasal en que se produce un cambio de la impedancia acústica. Los datos de mayor interés son las "áreas de sección transversal mínimas I y 2" (MCA I y MCA2, en inglés). MCAI corresponde anatómicamente al área a nivel de la válvula nasal (zona limitada por el borde caudal del cartílago alar superior y el tabique nasal), que es la zona de mayor resistencia 
en la nariz normal. MCA2 corresponde al área a nivel de la cabeza del cornete inferior. Al igual que la RMMAA, el estudio se puede realizar antes y después del uso de vasoconstrictor con la misma finalidad y similar interpretación ${ }^{(4,5)}$.

\section{Nasofaringolaringoscopía (NFL)}

Este examen permite evaluar la anatomía de toda la vía aérea superior, así como también la función del velo del paladar, el movimiento de las cuerdas vocales y el proceso de deglución. Se realiza con un fibroscopio flexible que se introduce por las fosas nasales, hasta observar faringe y laringe. Habitualmente se realiza en un paciente vigil al cual se le ha aplicado lidocaína tópica en las fosas nasales y, según el caso, vasoconstrictor (oximetazolina) en las mismas. Durante el examen se le puede solicitar al paciente que hable, que tosa o que degluta según lo que se quiera evaluar. Desde el punto de vista anatómico se debe evaluar: Presencia de desviaciones del tabique nasal, tamaño de los cornetes inferiores, presencia y tamaño del tejido adenoideo, cantidad y calidad de la secreción nasal, tamaño de las amígdalas palatinas y de la base de la lengua y su relación con la cavidad orofaríngea, abducción de las cuerdas vocales, amplitud de la subglotis, y presencia a cualquiera de estos niveles de masas o deformidades patológicas

Para evaluar la función del velo del paladar, se observa si su movimiento permite el correcto cierre del esfínter velofaríngeo tanto en la deglución como en la emisión de fonemas nasales como las letras "M" o "N". Para esto, se posiciona la fibra en el tercio posterior de la fosa nasal y se pide al paciente que emita los fonemas en cuestión o degluta para valorar el escape anormal de aire o el paso de alimentos hacia la rinofaringe, ambos signos de insuficiencia velopalatina.

Para la evaluación de la motilidad cordal y la voz, se posiciona la fibra a nivel de la supraglotis y se solicita al paciente que emita distintas vocales, a distintas intensidades y alturas (graves o agudas). El movimiento cordal en condiciones normales debe ser simétrico, al igual que la altura cordal y la tensión al fonar. Podrían observarse vicios o compensaciones fonatorias, como la aducción patológica de las bandas ventriculares (Figura I). De este modo podremos diagnosticar parálisis de cuerdas vocales, alteraciones del nervio laríngeo superior, disfunciones de tipo musculotensionales, etc. Además se debe evaluar detalladamente la superficie de las cuerdas vocales en busca de lesiones estructurales que pudieran generar disfonía, como nódulos, quistes o pólipos.

La sensibilidad laríngea también se puede evaluar a través de este examen, tocando con la fibra óptica distintos puntos de la laringe, lo que en condiciones normales genera en el paciente algún movimiento reflejo ya sea cierre glótico, tos o arcadas. Para evaluar el mecanismo de deglución el paciente debe estar sentado, idealmente vigil y cooperador. Previamente se debe observar la anatomía y sensibilidad laríngeas, así como la motilidad cordal. Luego, con la fibra óptica entre el velo del paladar y la epiglotis, se ofrece al paciente alimentos de diferentes consistencias y volúmenes (papilla, jalea, galleta, jugo, etc.). Se consideran anormales los siguientes hallazgos: presencia del bolo alimenticio por más de 2 segundos en hipofaringe sin gatillar deglución, persistencia de alimento en hipofaringe luego de ocurrida la deglución, penetración laríngea del alimento (paso de éste al vestíbulo laríngeo, sin traspasar las cuerdas vocales), aspiración del alimento (paso de éste a la tráquea a través de las cuerdas vocales), regurgitación del alimento desde el esófago hacia la hipofaringe (Figura 2). La deglución también puede ser evaluada con una videofluoroscopía, examen que se considera complementario a la evaluación de la deglución por nasofaringolaringoscopía ${ }^{(6)}$.

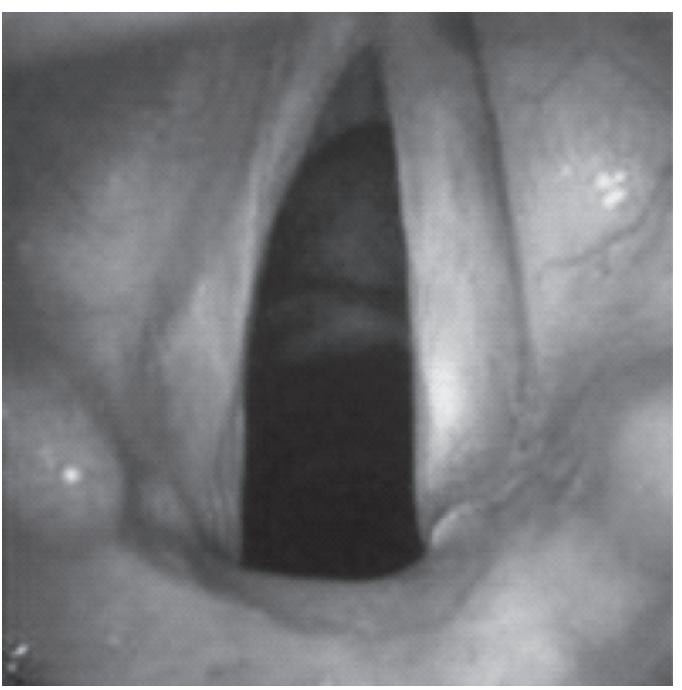

Figura I. Parálisis de cuerda vocal izquierda en posición lateral. Al fonar sólo la cuerda vocal derecha se aduce, la izquierda permanece abducida.

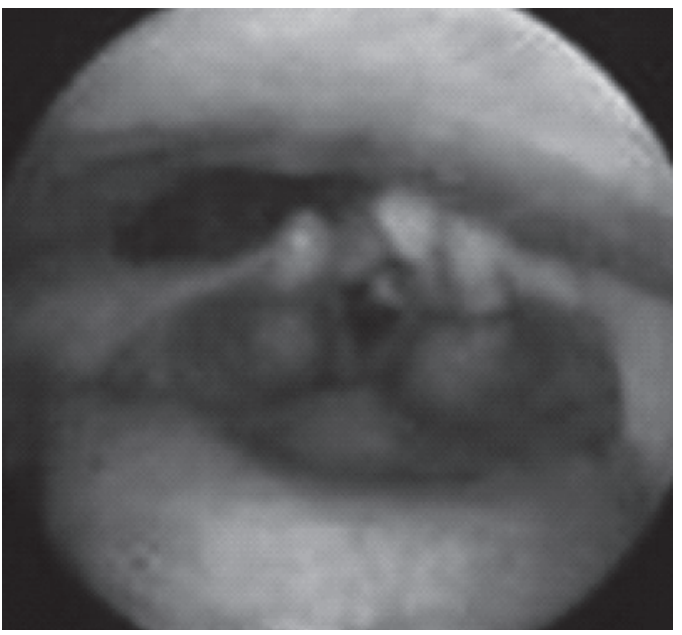

Figura 2. Estudio de deglución alterado, con retención de alimento en hipofaringe, penetración laríngea y aspiración hacia vía aérea inferior.

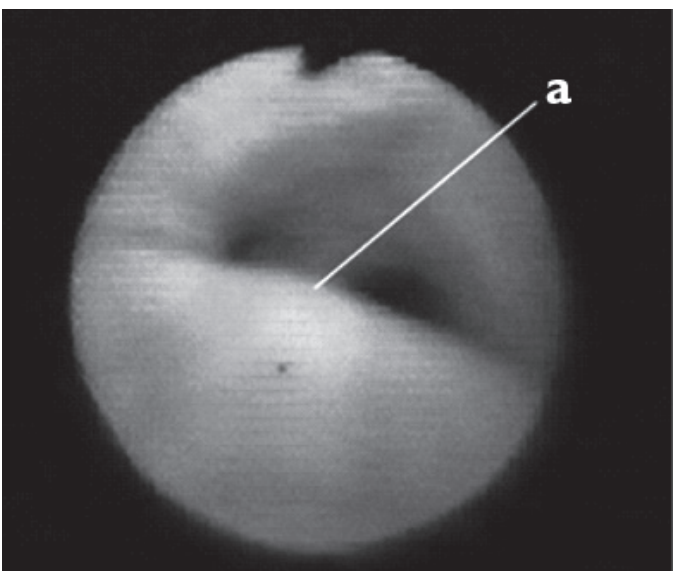

Figura 3. Traqueomalacia: Disminución significativa del lumen traqueal, visión endoscópica. (a) pares posterior traqueal. 


\section{Vía aérea central}

\section{Broncoscopía}

Es el standard de referencia actual, siendo superior la flexible con respecto a la rígida, ya que el paciente respira espontáneamente, $y$ en algunos casos se le puede solicitar que realice respiraciones profundas, exhalaciones forzadas y maniobras de tos, para lograr observar si existe colapsabilidad de la vía aérea (Figura 3). Sus defectos son la subjetividad del diagnóstico, ya que este va a depender de la experiencia del operador y por otro lado en la mayoría de los casos la dinámica de la vía aérea es afectada por la presencia del instrumento, presiones de ventilación, sedación o anestesia ${ }^{(7,8)}$.

\section{Imágenes}

Debe considerarse los riesgos asociados a cada procedimiento, tales como la necesidad de anestesia, intubación, irradiación y uso de medios de contraste. La tradicional radiografía simple de tórax realizada al final de la espiración no es un buen método para evaluar adecuadamente la colapsabilidad de la vía aérea. Al combinar las radiografías inspiratoria y espiratoria para el diagnóstico de traqueobroncomalacia se encontró una sensibilidad de 62\%. Hasta hace algunos años se usaba la cine- fluoroscopia, que mostraba una especificidad de 94-100\% para el diagnóstico de traqueobroncomalacia, teniendo las ventajas de no ser invasivo, no requerir colaboración por parte del paciente,y en los lactantes permitir la evaluación dinámica de la vía aérea durante el llanto y la alimentación. Sus desventajas son que no se visualiza con exactitud los detalles anatómicos traqueales, no se puede mostrar en forma simultánea las paredes anterior y posterior de la tráquea y la interpretación es subjetiva. La broncografía actualmente es un método es desuso por ser invasiva, requerir anestesia y administración de medio de contraste dentro de la vía aérea ${ }^{(6,7)}$.

La tomografía computada helicoidal con multicorte y reconstitución en 3D es capaz de adquirir datos de volumen de la vía aérea al final de la inspiración y durante la espiración activa. Se consideran alteración los casos en los cuales el calibre de la vía aérea disminuye en $50 \%$ o más. Se ha encontrado una buena correlación entre este concepto y el hallazgo de traqueobroncomalacia mediante fibrobroncoscopia. El gran defecto de este método es la alta irradiación ${ }^{(7,9)}$.

Existen estudios preliminares que muestran un potencial rol de la resonancia nuclear magnética dinámica para la evaluación de traqueomalacia solicitando que el paciente tosa, y para la evaluación de pacientes con apnea obstructiva del sueño(9).

\section{Curva flujo/volumen (Figura 4)}

La curva flujo/volumen obtenida por espirometría forzada es característica según el segmento de la vía aérea central que esté comprometido, y ayuda a distinguir entre una obstrucción variable intratorácica, extratorácica, o una obstrucción fija de la vía aérea central ${ }^{(1,11)}$.

En el caso de existir una alteración dinámica de la tráquea intratorácica o bronquios mayores se observa un flujo máximo inicial que refleja una rápida expulsión de un pequeño volumen de aire, siguiendo esta fase se ve una caída sostenida del flujo, con forma semejante a una meseta, traduciendo exhalación de escasa cantidad de aire,y luego a flujos espiratorios bajos recuperar la velocidad, hasta finalizar la espiración a volumen residual. Los valores de VEF, y CVF en general no están alterados, pero sí lo está el valor del FEM (flujo espiratorio máximo). La fase inspiratoria de la curva estará normal (patrón de obstrucción variable intratorácico) ${ }^{(8,10)}$.

En las situaciones de obstrucción dinámica de la vía aérea central extratorácica, se verá afectada la fase inspiratoria de la curva flujo/volumen, siendo normal la fase espiratoria (patrón de obstrucción variable extratorácica). En los casos de obstrucción fija, mecánica, de la vía aérea central intra o extratorácica, ambas fases de la curva se verán de forma aplanada (patrón de obstrucción fija) ${ }^{(10)}$.

\section{Vía aérea periférica}

\section{Espirometría}

La espirometría forzada es el examen más simple para evaluar la vía aérea periférica, mediante el análisis de la fase espiratoria de la curva flujo/volumen y los valores obtenidos en volúmenes y flujos espiratorios forzados. A medida que progresa la espiración forzada, la retracción elástica pulmonar y el calibre de la vía aérea, que dependen del volumen pulmonar, disminuyen progresivamente, hasta que en algún punto del árbol bronquial se iguala la presión dentro y fuera de la vía aérea ("punto de igual presión"). Si este se ubica en una zona colapsable del árbol bronquial, se produce una limitación dinámica al flujo aéreo, y a partir de ese momento el flujo de aire no depende del esfuerzo. Si un paciente está

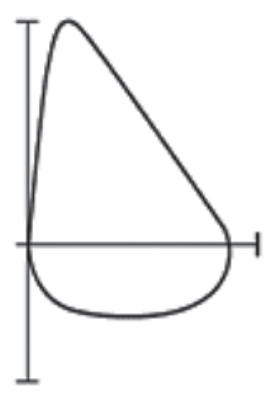

A Obstrucción variable extratorácica

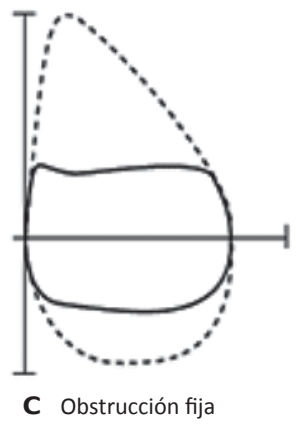

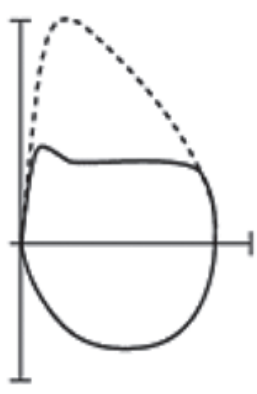

B Obstrucción variable intratorácica

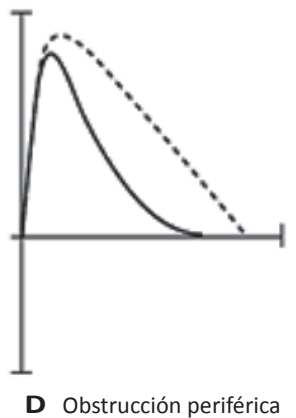

Figura 4. Espirometría: curvas flujo/volumen. 
cursando una enfermedad obstructiva, por ejemplo una crisis asmática, este punto de igual presión se traslada hacia distal, las vías aéreas se cierran precozmente, la curva volumen/ tiempo no presentará meseta y la curva flujo/volumen tendrá aspecto cóncavo. Según la severidad del compromiso inicialmente se comprometen los flujos espiratorios forzados $\left(\mathrm{FEF}_{25-75}\right.$ y $\left.\mathrm{FEF}_{50}\right)$, y luego volúmenes espiratorios $\left(\mathrm{VEF}_{1}\right.$ y en casos más severos CVF, con relación $\mathrm{VEF}_{\mathrm{I}} / \mathrm{CVF}$ disminuida) (Figura 4) $)^{(1-13)}$.

La espirometría forzada también es el método más utilizado para realizar la prueba de respuesta a broncodilatador, con el fin de evidenciar la presencia de obstrucción bronquial y valorar reversibilidad. Otros exámenes usados con este mismo objetivo son la medición de resistencia de la vía aérea, oscilometría de impulso, resistencia interrumpida y medición de volúmenes pulmonares estáticos ${ }^{(12,14)}$. En pacientes escolares y adolescentes se considera significativo un aumento en el $V E F$, en $12 \%$ con respecto al $V E F$, basal. El aumento en flujo espiratorios $\left(\mathrm{FEF}_{25-75}\right)$ sólo es de valor cuando la CVF basal y post-broncodilatador son semejantes. En pacientes en edad pre-escolar aún no se ha establecido la magnitud del cambio en $\mathrm{VE}_{0,5} \circ \mathrm{VEF}$, para ser considerado como significativo. $\mathrm{A}$ toda edad es de gran utilidad constatar un cambio en la forma de la curva flujo/volumen espiratoria ${ }^{(12,13)}$.

Otra forma de evaluar la función de la vía aérea usando la espirometría es demostrar la presencia de hiperreactividad bronquial, característica, aunque no exclusiva, del asma bronquial. Para esto existen pruebas de provocación bronquial con estímulos directos e indirectos. Los primeros actúan sobre las células efectoras responsables de la broncoconstricción (músculo liso, endotelio, células secretoras): metacolina, histamina, manitol. El segundo grupo actúa sobre células neuronales y mastocitos, los cuales en un segundo paso estimulan las células efectoras: son estímulos fisicos como el ejercicio, solución hipertónica, aire frío, hiperventilación, y estímulos químicos como adenosina, alergenos ambientales.

No se explicará el detalle de cada uno de estos exámenes. Los estímulos más utilizados en nuestro medio son la metacolina y ejercicio, destacando el alto valor predictivo negativo para el diagnóstico de asma bronquial de la provocación bronquial con metacolina, y la alta especificidad pero baja sensibilidad de la provocación con ejercicio( ${ }^{(1,15)}$.

\section{Resistencia de la vía aérea}

La resistencia de la via aérea (Raw) depende de varios factores: tipo de flujo, fricción del aire contra las paredes, calibre de la vía aérea, volumen pulmonar y presión transmural. El único método disponible para medir directamente la resistencia de la vía aérea es la pletismografía corporal, motivo por el cual es considerado el gold standard. Se registra la presión alveolar y el flujo aéreo durante varias maniobras de jadeo, calculándose la resistencia total de la vía aérea. Es posible utilizar esta técnica desde el período de lactante, bajo sedación $n^{(1,11)}$

Los otros métodos disponibles tienen la ventaja de requerir poca colaboración, siendo posible su realización desde edad pre-escolar. Sin embargo, tienen la desventaja de no medir resistencia de la vía aérea como tal, ni ser tan altamente reproducibles como las mediciones de Raw(II).
En la oscilometría de impulso se aplica una onda externa de sonido en la boca (a frecuencias entre 4 y $20 \mathrm{~Hz}$ ) y la relacion presion/flujo resultante se mide como impedancia. Esta refleja el impedimento al flujo en el sistema respiratorio, y es la suma de la resistencia (asociada a pérdidas friccionales en la vía aérea y parénquima pulmonar) y reactancia pulmonar (recíproco de la compliance pulmonar)(II).

Otra forma de medir resistencia es la técnica de resistencia interrumpida (Rint). Esta asume que al momento de la interrupción transitoria del flujo en la vía aérea la presión en la boca es igual a la presion alveolar. La Rint se calcula según la presión y flujo en la boca medidos inmediatamente antes de la oclusión. La Rint además de medir presión en vía aérea toma en cuenta la presión de retracción elástica del tejido pulmonar y pared torácica(II).

\section{CONCLUSIÓN}

La prevalencia de enfermedades que comprometen la vía aérea en pacientes pediátricos es alta e implica una diversidad de diagnósticos, que determinan pronósticos también variables. Actualmente, se cuenta con métodos especíícos, confiables y seguros para evaluar la anatomía y funcionalidad de la vía aérea, lo que permite optimizar el tratamiento y evolución de cada paciente.

\section{REFERENCIAS}

I. Lumb A. Functional Anatomy of the Respiratory Tract. Nunn's Applied Respiratory Physiology. Fifth Edition. Ed ButterwothHeinemann 2003. Chapter 2. p I5-34.

2. Levitsky M. Function and Structure of the Respiratory System. Pulmonary Physiology. Sixth Edition. Ed McGraw-Hill 2003. Chapter I. p I- 10.

3. Cole P, Fenton RS. Contemporary rhinomanometry. J Otolaryngol 2006; 35: 83-7.

4. Haavisto LE, Vahlberg TJ, Sipila JI. Reference values for acoustic rhinometry in children at baseline and after decongestion. Rhinology 201 1; 49: 243-7.

5. Finkelstein A, Lara C, Faba G, Caro J. Rol de la rinometría acústica en la evaluación de los pacientes candidatos a rinoseptoplastía. Rev Otorrinolaringol Cir Cabeza Cuello 2009; 69: 109- 16.

6. Hiss SG, Postma GN. Fiberoptic endoscopic evaluation of swallowing. Laryngoscope 2003; I 13: 1386-93.

7. Boiselle P, Ernst A. State-of-the-Art Imaging of the Central Airway. Respiration 2003; 70: 383-94.

8. Murgu S, Colt H. Tracheobronchomalacia and excessive dynamic collapse. Respirology 2006; 1 I : 388-406.

9. Zhi Yie Tan J, Ditchfield M, Freezer N. Tracheobronchomalacia in children:review of diagnosis and definition. Pediatr Radiol 2012; 247: 2367-75.

10. Levitsky M. Mechanics of breathing. Pulmonary Physiology. Ed Mc Graw Hill Sixt Edition 2003. Chapter I. p I I-53.

II. González Pérez-Yarza E, Aldasoro Ruiz A, Korta Murua J, Mintegui Aramburu J, Sardón Prado O. Volúmenes, flujos, resistencias. La Función Pulmonar en el Niño. Principios y Aplicaciones. Ed Ergon 2007 p 23-37. 
12. Miller MR, Hankinson J, Brusasco V, Burgos F, Casaburi R, et al. Standardization of spirometry. Eur Respir J 2005; 26: 31 9-38.

13. Pellegrino R, Viegi G, Brusasco V, Crapo RO, Burgos F, Casaburi $\mathrm{R}$, et al. Interpretative strategies for lung function tests. Eur Respir J 2005; 26 (5): 948-6.
14. Shim C. Response to bronchodilators. Clin Chest Med 1989; 10: I55-16.

15. American Thoracic Society. Guidelines for exercise and methacholine challenge testing- 1999. Am J Respir Crit Care Med 2000; |61: 309-29. 